\title{
Personalized and Precision Medicine (PPM) as a Unique Advanced Frame of Healthcare to Secure the National Biosafety and Longevity
}

\section{Veronika Medvedeva ${ }^{1}$, Anton Yuryev ${ }^{8}$, Hiroyuki Abe $^{9}$, Andrey Svistunov ${ }^{1}$, Andrew D Miller ${ }^{11-14}$, Maria Studneva ${ }^{1}$, Eiji Matsuura ${ }^{15}$, Elena Antonova ${ }^{1,7}$, Vassiliy Tsytsarev ${ }^{10,11}$ and Sergey Suchkov ${ }^{1-6 *}$}

${ }^{1}$ Sechenov University, Moscow, Russia

${ }^{2}$ A.I. Evdokimov Moscow State University of Medicine and Dentistry, Russia ${ }^{3}$ EPMA, Brussels, EU, Belgium

${ }^{4} \mathrm{PMC}$, Washington, $D C$, USA

${ }^{5}$ ISPM and Abe Cancer Clinic, Tokyo, Japan

${ }^{6}$ AHA, Houston, TX, USA

${ }^{7}$ Municipal N.F. Filatov's Clinical Pediatric Hospital, Russia

${ }^{8}$ Center for Bioinformatics, Elsevier, Rockville, MD, USA

${ }^{9}$ ISPM and Abe Cancer Clinic, Tokyo, Japan

${ }^{10}$ Fischell Center of Bioengineering, University of Maryland, College Park, USA

${ }^{11}$ Department for Neuronal Networks, John Hopkins University, MD, USA

${ }^{12}$ Veterinary Research Institute Czech Republic

${ }^{13}$ Mendel University, Brno, Czech Republic

${ }^{14}$ Shanghai Jiao Tong University, Shanghai, PRC

${ }^{15}$ Okayama Medical Innovation Center (OMIC) and Collaborative Research Center for

OMIC, Okayama University Graduate School of Medicine, Dentistry and Pharmaceutical

Sciences, Okayama, Japan

*Corresponding Author: Sergey Suchkov, Sechenov University, Moscow, Russia.
Received: April 19, 2021

Published: May 20, 2021

(C) All rights are reserved by Sergey Suchkov., et al.

\section{Abstract}

Policy formation in the field of individual health promotion and protection is leading task of each state. The up-dated medicine is Personalized and Precision Medicine (PPM), by improving public health and supporting longevity. In this regard, an upgraded healthcare service, which includes the philosophy, concept and equipment of PPM and aimed at identifying the disturbance at its subclinical stage, is being created and rooted. PPM focuses on predictive and preventive measures that contribute to the development of individualized program for managing a healthy lifestyle that reduce the morbidity rates and increase the working capacity of the population. To succeed the goals of value-based healthcare and the implementation of the PPM principles, it is necessary to combine the assets of the newest advances in basic science with clinical medicine, followed by the introduction and promotion of new generation's translational applications.

Keywords: Personalized and Precision Medicine (PPM); Artificial Intelligence; Healthcare

\section{Introduction}

Over the course of history, healthcare and thus healthcare philosophy have been focused predominantly on efforts to probe the already diseased individual by focusing down on a type of disorder (nosology) rather than on health or so-called pre-illness conditions. Much less effort has been placed on keeping individuals

Citation: Sergey Suchkov., et al. "Personalized and Precision Medicine (PPM) as a Unique Advanced Frame of Healthcare to Secure the National Biosafety and Longevity". Acta Scientific Microbiology 4.6 (2021): 82-84. 
from developing disorders in the first place. PPM is expected to transform this situation giving healthcare professionals of tomorrow much more reliable control over morbidity, mortality and disabling rates, and significantly optimize the cost and efficacy of treatment for those who have fallen ill and already diseased, or are still persons-at-risk. PPM is a name for the grand new paradigm in healthcare management being based first on prevention, pre-clinical detection of the illness, and delivery of drugs to target tissues with exceptional levels of precision.

\section{The grand challenge of advanced healthcare model}

Development trends of fundamentally new type of practical health care (personalized medicine) dictate new healthcare requirements. As regard scientific platform, by bringing together post-genome sciences and nano-technological platforms, such as Bioinformatics and Artificial Intelligence (AI), in one strong unit, translational medicine (TraMed) is the main resource for creation, introduction and promotion of the new generation's translational applications. In addition, translational medicine is a concept that solves the challenges of the future using the achievements of fundamental developments directly in the format of new pharmaceutical designs as part of the PPM strategy.

PPM is highly dependent upon biomarkers, molecular health indicators, that can be used to enhance diagnosis in order to help to predict treatment and pharmacological responses. Currently, the principal priority of PPM and TraMed as the global tool of the latter is to find potential and highly informative biomarkers with their subsequent selection. PPM as a concept and TraMed as a unique armamentarium to get the latter re-armed would concern the adjustment of treatments to individual or subgroups of patients based on the use of disease-specific biomarkers. There continues a great debate as to the overall success of this personalized process to identify working biomarkers or their complexes in order to design effective treatment programs.

The challenge now faced by the diagnostics and pharmaceutical industries is to maintain and preferably increase the rate at which novel biomarkers can be discovered, qualified as having clinical value, translated into validated assays and, ultimately, introduced into routine use.

At the same time, it must be remembered that the greatest achievement of TraMed is the identification of biomarkers patho- genesis. Consequentially, such diagnostics, based on molecular pathological indicators, can secure both a more accurate illustration of the health status of a particular patient or a person-at-risk, and to develop a specific drug with the proper dose of the active substance, with potential benefits of increased clinical efficacy and individual safety. Ultimately, this will lead to the development of better targeted therapies and deliver PPM to finally bring about individualized patient management in a wider range of conditions.

Using statistics data, more than 9,5 million people are diagnosed with chronic disease every year. Biomarkers are therefore becoming invaluable tools for chronic disease detection, diagnosis, prognosis and treatment selection. These can also be used to predict the malignancy, to identify the initial stage, to localize the tumor and determine its stage and next steps of pathology. Identification of such signature in surrounding cells or at more distal and easily sampled sites of the body can also influence the management of disease.

\section{Biomarkers as a unique unit of PPM model}

As well as know, PPM provides procedures for disease prediction and for the prediction of consequences and complications. It should be noted that these arrangements are more suitable in healthy persons than in patients with a disease manifestation. In this regard, the biomarker-based analysis is intended as a first step towards a more personalized and precision treatment.

Biomarkers are essential for the development of PPM. Biomarkers can be used in drug development (for example, patient selection) or in clinical practice, such as determining which drugs are the best fit for patients, depending on the presence or absence of certain biomarkers. The application of biomarkers to improve patient outcomes is now common in clinical trials, which is rapidly being adopted into daily medical practice. Biomarkers can be anything that can be measured in a person (a patient or a personat-risk) and can range from genome, proteome, metabolome measured from blood, tissue and other biomaterials to imaging such as digital pathology and radiology with special contrast agents.

For instance, a diagnostic biomarker is applied daily to identify individuals with a disease or condition of interest or to define a subset of the disease. A prognostic biomarker is used to estimate the outcome for a patient in the absence of a treatment. A predictive biomarker is used to monitor the effectiveness of prescribed 
treatment. Ideally a predictive biomarker that measures mechanisms of sensitivity or resistance would be used along with the prognostic biomarker to select the correct chemotherapy for the patient's specific tumor.

The ability to measure biomarkers in blood and tissue samples to provide pre- (including subclinical) detection of disease, measurements for treatment efficacy, and disease progression for a host of chronic disorders has been critical to rapid advancements in PPM-based health and to helping researchers improve a drug's impact.

Biomarkers can be used in basic, translational, and clinical research as drug development tools, in clinical settings to facilitate drug re-purposing and inform patient care decisions, and can be incorporated into drug development through the drug approval process, scientific community consensus followed by regulatory acceptance, and biomarker qualification. The academic, clinical and industrial communities, patients, care givers and providers, patient advocacy organizations, biopharma and biotech, and regulators all play important roles in the development and application of biomarkers.

Current (canonical) biomarkers used in health care and health research are based on a comprehensive assessment of diagnosis of the pathogenesis initial stages and its progression. A new generation of biomarkers is required that qualitatively define all aspects of the variability of unified system indicators, opening a new way to optimize treatment and rehabilitation costs and expanding the possibilities for building new products and services in this area.

Next-generation biomarkers (NGBs)) are associated with determining the probability of developing chronic pathologies or cancer-predisposed condition. So, the key factors contributing to the growth of the global NGBs market include high prevalence of chronic diseases (predominantly, cancer), high government funding for cancer research and clinical trials, rising adoption of biomarker for diagnostic applications, increasing application in drug discovery and development. Introduction of new technologies such as digital biomarker and rising adoption in PPM drive the market for NGBs.

For instance, the biomarkers of the future would have to be used for: (i) screening the general population or individuals at risk (panels of screening and predisposition biomarkers); (ii) the detection of the presence of a particular type of cancer (panels of di- agnostic and prognostic biomarkers); (iii) monitoring the progression of the disease, and predicting the tumor's out-come (panes of prognostic biomarkers); (iv) understanding whether a patient will benefit from a specific drug treatment (panels of predictive biomarkers); ( $v$ ) evaluating the drug's efficacy and optimizing the treatment, providing the tool to tailor treatment for individual cancer patients or persons-at-risk (panels of pharmacodynamics biomarkers) [1-3].

\section{Conclusion}

The main task of PPM is to extend healthy life and increase the size of working-age population, with simultaneous and timely detection of pathological changes in the body, and targeted measures aimed at preventing diseases. Implementation of PPM requires a lot before the current model "physician-patient" could be gradually displaced by a new model "medical advisor-healthy person-atrisk". This is the reason for developing global scientific, clinical, social, and educational projects in the area of PPM to elicit the content of the new branch.

Based on an interdisciplinary basis, PPM is generating academic and clinical profit that can be effectively used in the daily practice in the future to come. No less important is that such a multi-disciplinary alliance should be properly and correctly structured and adopted under real real-world environment. Moreover, academyrelated, clinical and biopharma communities as the Holy Trinity in consolidating global efforts to make the model of PPM re-armed and open to be promoted as a new advanced model of healthcare service to secure the National and International Biosafety.

\section{Bibliography}

1. TA Bodrova., et al. "Introduction into PPPM as a new paradigm of public health service: an integrative view". EPMA Journal 3.16 (2012): 3-16.

2. Miller A D. "Nanomedicine therapeutics and diagnostics are the goal". Therapeutic Delivery 7.7 (2016): 431-456.

3. Lejbkowicz I., et al. "Participatory medicine and patient empowerment towards personalized healthcare in multiple sclerosis". Expert Review of Neurotherapeutics 12.3 (2012): 343352.

\section{Volume 4 Issue 6 June 2021 (C) All rights are reserved by Sergey Suchkov., et al.}

